\title{
UN ENFOQUE CLADISTA PARA LA INSERCIÓN DE FÓSILES EN LA FILOGENIA
}

\author{
Axel P. Retana-Salazar \\ Centro de Investigación en Estructuras Microscópicas (CIEMic), Ciudad de la \\ Investigación, Universidad de Costa Rica 2060 \\ axel.retana@ucr.ac.cr
}

(Recibido: 23/09/08 ; aceptado: 12/12/08)

\begin{abstract}
The study of fossils is one of the most important fields of biology and geology. However is difficult to insert these forms in phylogenies of modern organisms. This paper proposes a method for inserting fossil forms in cladograms that describe phylogenetic scenarios of recent life forms. Three possible hypothetical scenarios have been considered for the development of this methodology. The method consist in four steps, 1) the phylogenetic reconstruction of the modern forms with the current characters used for the group, 2) the inclusion of the most complete fossil form in the data matrix without the characters coded as "?", 3) the analytical position of the taxa in the topologies (if there are more than one) using the strict consensus or majority rule consensus, if the first do not resolve any possible group, 4) the inclusion of the fossil form in the position where is most probably inserted with the characters exhibited. A real case has been used to illustrate the validity of this method. The inclusion of fossils with this method shows that the state of preservation of the fossil is not necessarily a good indicator of the importance of the fossil form.
\end{abstract}

Key words: Fossils, phylogenetics, cladistics.

RESUMEN: El estudio de los fósiles es uno de las áreas de mayor importancia de la biología y la geología. Sin embargo es difícil la inserción de estas formas en las filogenias de organismos modernos. Este documento propone un método para insertar las formas fósiles en los cladogramas que describen los escenarios filogenético de las formas de vida actuales. Se consideran tres posibles escenarios hipotéticos para el desarrollo de esta metodología. El método consisten en cuatro pasos, 1) efectuar la reconstrucción filogenética de las formas modernas con los caracteres actuales utilizados para el grupo, 2) se efectúa la inclusión de los fósiles empezando por la forma más completa en la matriz de datos sin incluir los caracteres codificados como “?”, 3) se analiza la posición de los taxones en las diferentes topologías (si hay más de una) con un consenso estricto o un majority rule consensos si el anterior no logra resolver ninguna agrupación, 4) se efectúa la inclusión del fósil en la posición de mayor probabilidad según los caracteres exhibidos. Se utiliza un caso real para ilustrar la validez del método y su aplicabilidad a contextos reales. La inclusión de formas fósiles con este método muestra que el estado de preservación de los fósiles no es necesariamente un indicador de la importancia de estos en el esclarecimiento de las relaciones filogenéticas.

Palabras clave: Fósiles, filogenia, cladística. 


\section{INTRODUCCIÓN}

Una de las evidencias más contundentes de que el proceso evolutivo es real es el hallazgo de formas preservadas en los diferentes estratos geológicos (Mayr 2001, Bravo-Cuevas y GonzálezRodríguez 2007). Una evidencia de esto es que los primeros esquemas evolutivos fueron propuestos por paleontólogos (Matthew 1926, Stirton 1940, Simpson 1951). Derivado de estos enfoques en los que se utilizaban tanto las formas fósiles como las recientes se estableció la existencia de una relación ancestro-descendiente (Bravo-Cuevas y González-Rodríguez 2007), la cual es fundamental para comprender los procesos evolutivos. Dicha relación ha sido motivo de estudio e incluso existen propuestas matemáticas acerca de su interpretación (Retana-Salazar y Retana-Salazar 2004). De estas argumentaciones fundamentadas en estudios de grupos fósiles se derivó el pensamiento de algunos especialistas que sugirieron que los fósiles proporcionan la base sólida de la clasificación evolutiva (Simpson 1951). Por su parte, otros biólogos han considerado que la evidencia fósil es incompleta y por ende proporciona muy poca información para el establecimiento de relaciones filogenéticas (Hennig 1966, Nelson 1978, Patterson 1981, Ax 1987). Sin embargo, estudios de filogenia con aplicación de la cladística han demostrado que los grupos en los que se incluyen tanto formas fósiles como actuales han demostrado lo contrario, siendo los fósiles de gran utilidad en la determinación de puntos históricos importantes como el punto de origen de un grupo y su punto de diversificación, y estos fósiles también han contribuido al esclarecimiento de la polaridad de los estados de carácter (Gauthier et. 1988, Forey et al. 1994, Bravo-Cuevas y GonzálezRodríguez 2007).

Por otra parte, los grupos fósiles pueden ser de gran ayuda en la calibración y prueba de sistemas de reconstrucción filogenética, en los cuales se necesita de un patrón, que pueda ayudar en la determinación de las posibles relaciones evolutivas entre organismos (Retana-Salazar y RetanaSalazar 2008). Por todas estas razones negar la importancia medular de incluir las formas fósiles en los estudios de filogenia es solo negar el papel preponderante de comprender las formas de vida que existieron para entender aún mejor las actuales. Siendo la evolución un estudio de origen histórico y que por ende no muestra reversa (Lotka 1956, Brooks y Wiley 1986, Retana-Salazar y Retana-Salazar 2008), es indispensable entender el pasado para una correcta interpretación actual.

El estudio de evidencia fósil, hasta el momento, depende en mucho de la cantidad de especimenes hallados. Si se tiene la suerte de encontrar un yacimiento de fósiles con múltiples fósiles de una determinada especie, la descripción del mismo suele ser muy detallada, como ha sucedido con los fósiles del Mioceno Aragonés (Peñalver 1998). La inserción de los fósiles en las filogenias ha dependido del hallazgo de al menos una apomorfía que justifique su inclusión dentro del modelo evolutivo escogido para la interpretación del fósil (Forey 1994). Esta forma de estimación ha llevado a numerosos errores de interpretación y de clasificación., como ha sucedido recientemente con la inclusión de ciertos fósiles de ácaros oribátidos en el grupo de los piojos (Phthiraptera)(Dalgleish et al 2006). La evaluación de los caracteres en ausencia de herramientas más objetivas que el propio criterio puede causar sesgos insospechados a la interpretación de la misma. Por eso la inclusión de fósiles en filogenia debe obedecer a una metodología repetible, que es lo que se intenta proponer en este trabajo.

\section{EL PROBLEMA DE LA CODIFICACIÓN FÓSIL}

Uno de los mayores problemas de la evidencia fósil en su uso con los actuales métodos de inferencia filogenética, es la ausencia de caracteres en los restos conservados (Forey 1994), los cuales al estar ausentes causan problemas a la resolución filogenética establecida (Platnick et 1991). Por esta razón, en este trabajo se propone una alternativa de exploración metodológica que permite una mayor aproximación de la posición más probable que tuvieron los fósiles con respecto a las formas actuales en los estudios de filogenia. Esto puede ser de gran utilidad en la inferencia de la posición de los grupos fósiles, y derivado de ello nuevas interpretaciones de los caracteres considerados por los neontólogos como de importancia en filogenia. 
Debido a que los fósiles pueden ser muy incompletos muchos caracteres deben de codificarse como "?" al incluirlos en una matriz de datos junto con formas actuales. El uso de taxa con muchos caracteres codificados como "?" produce serios problemas de resolución (Forey 1994). Al establecer una matriz de caracteres en la que se combinan formas fósiles y actuales no solo es pertinente considerar los caracteres de los que no hay evidencia en las formas fósiles, sino también el problema de los caracteres presentes en algunos fósiles que no se hallan en ninguna forma actual (Forey 1994). En este caso, desde la perspectiva metodológica cladista estos caracteres deben codificarse como "no aplicable", con lo que los grupos actuales pueden codificar para estos caracteres como "caracteres perdidos" (Forey 1994).

Para los algoritmos computacionales actuales hay dos opciones de reconstrucción: la planteada por el algoritmo de Hennig86 y la planteada por el algoritmo PAUP. La mayoría de los actuales programas de explicación y reconstrucción filogenética para equipos PC son modificaciones del algoritmo clásico utilizado por Hennig86. Mientras que los sistemas MacIntosh utilizan el algoritmo PAUP. Platnick y colaboradores (1991) demostraron que para estos algoritmos computacionales las alternativas "perdido" o "no aplicable" son tratados de la misma forma, de manera que estas codificaciones permiten al algoritmo escoger estados alternativos, lo que produce resoluciones filogenéticas falsas. Retana-Salazar y Retana-Salazar (2008) han planteado que este problema puede ser solventado presentando una primera reconstrucción filogenética utilizando solo aquellos caracteres presentes en todos los taxa y luego introduciendo los caracteres con menor información en las posiciones probabilisticas más acertadas.

\section{MATERIAL Y MÉTODO}

Se utilizó el programa PAUP 3.1.1 para la reconstrucción filogenética. Se utilizaron tres matrices hipotéticas de datos, para estudiar las variaciones obtenidas en la resolución filogenética, con el fin de simular diferentes situaciones posible a la hora de analizar la posición de fósiles dentro de un arreglo filogenético.
Las dos primeras evalúan dos posibles entornos evolutivos que se hallan documentados en el registro fósil. Se determinó el comportamiento de las matrices hipotéticas para cada caso dentro de una hipótesis filogenética resuelta mediante la técnica cladística. La tercera estudia el comportamiento cuando existen varios taxa fósiles en una matriz de datos.

Características de las matrices hipotéticas. Se generan tres matrices hipotéticas constituidas por 10 taxa y 20 caracteres. Las dos primeras matrices (1 y 2) presentan 9 taxa con la codificación completa para todos los caracteres, mientras que un taxón la presenta incompleta, codificada mediante "?". Cada matriz estudiada obedece a un diferente proceso evolutivo. La tercer matriz (3) presenta la inclusión de tres taxa incompletos donde dos mantienen la misma codificación y un tercero con una codificación incompleta diferente, es decir fósiles que exhiben diferente preservación de características.

Por último, se presenta un ejemplo real para ilustrar la utilidad del método, para este fin se eligió el sistema exhibido en la matriz $\mathrm{N}^{\mathrm{o}} 1$, ya que de los dos posibles estudiados es el que se halla con mayor frecuencia en los datos del registro fósil. Se estima la ubicación de máxima probabilidad de los fósiles incluidos en este estudio.

\section{CARACTERÍSTICAS DE LAS MATRICES UTILIZADAS}

\section{Matriz 1}

Presenta un único taxón incompleto. La codificación hipotética de un grupo fósil en el cual no se hallan caracteres que no se registran en los grupos actuales.

Escenario evolutivo real. Esta situación es la más común en el registro fósil y se puede hallar en múltiples ejemplos desde el registro fósil humano hasta el registro fósil entomológico.

\section{Matriz 2}

Presenta dos o más taxa incompletos donde al menos uno es fósil y otro una forma actual. 
La codificación hipotética de un grupo fósil que presenta caracteres que se hallan ausentes en los grupos actuales.

Escenario evolutivo real: Esta situación se registra en los fósiles de grupos como los peces ciclóstomos (lampreas) como en otros grupos donde ha habido fuertes variaciones estructurales entre las formas fósiles conocidas y las formas actuales. En este caso los fósiles presentan caracteres referentes a la cobertura de escudos óseos que no se encuentran en las especies actuales.

\section{Matriz 3}

Presenta varias incorporaciones fósiles. Estas formas fósiles con diferentes caracteres y nivel de completitud con respecto a las formas actuales.

Se analiza la forma de inclusión de las diferentes formas fósiles en un único grupo de datos.

Análisis de Filogenia: Se utilizó la opción exhaustiva para la evaluación de la resolución filogenética. Es la más precisa de las metodologías de exploración existentes ya que analiza todas las posibles ubicaciones de cada uno de los taxa (Kitching 1994). No se puede utilizar con matrices mayores a 10 o 20 taxa dependiendo de la cantidad de caracteres utilizados (Arnedo 1999). En este caso las matrices escogidas son de 10x20 lo cual permite un análisis robusto con el fin de poder efectuar la mejor exploración posible. En matrices de mayores dimensiones es conveniente utilizar la búsqueda heurística (Arnedo 1999). Algunos programas solo presentan la opción de análisis heurístico, por lo que se trabajó con PAUP. Todos los caracteres se consideran como desordenados y se utiliza un grupo hipotético $\mathrm{X}$ como grupo externo para la reconstrucción filogenética en cada caso.

\section{Fundamento metodológico para la inclusión de fósiles}

Platnick et al. (1991) establecen a partir de análisis teóricos que las matrices con caracteres codificados como "?" producen en muchos casos posibles resoluciones filogenéticas falsas. Los algoritmos computacionales existentes ante la presencia de caracteres desconocidos, perdidos o que no aplican, toman valores nuevos para estas codificaciones. Retana-Salazar y Retana-Salazar (2008) proponen que los análisis de filogenia deben efectuarse eliminando los caracteres que se codifican como "?", de esta forma se obtiene una mejor resolución fundamentada en los datos conocidos y luego deben incorporarse los caracteres con las codificaciones no claras en las posiciones más probables. Sin embrago, esto no es sencillo ya que la matriz puede perder resolución al eliminar estos caracteres.

Este problema es particularmente común a los paleontólogos, ya que las formas fósiles a menudo son incompletas. De ahí que haya una necesidad de incorporar estas formas altamente informativas de los procesos evolutivos en los puntos de mayor probabilidad en una filogenia debidamente estructurada. Cuando se trabaja con pocos caracteres o el estado del fósil es muy completo es relativamente simple la inserción del mismo en una determinada topología. Sin embargo, en muchos casos aparecen en los fósiles caracteres que generan conflicto con respecto a los parentescos, o en su defecto, se presentan matrices con muchos caracteres que hacen difícil el estudio de la posición más probable del fósil con respecto a las formas actuales.

El método propuesto aquí consta de tres pasos para obtener un resultado de máxima verosimilitud para la inserción de los fósiles en la topología más probable, de ahí que los pasos a seguir son:

1) Analizar la matriz de datos con las formas actuales completas en su codificación para los caracteres seleccionados. Es decir excluyendo las formas fósiles incompletas.

2) Analizar la matriz de datos incluyendo las formas fósiles más completas con respecto a los caracteres seleccionados y definir el punto de mayor parentesco con las formas actuales. En este paso deben excluirse del análisis aquellos caracteres que se hallen codificados como desconocidos, perdidos o no aplicables. 
3) Tomar la posición más probable de la (s) forma (s) fósil (es) obtenida a partir del análisis del paso 2 y ubicar estas formas fósiles en la topología obtenida en el paso 1 .

\section{RESULTADOS}

\section{Análsis del caso 1}

Escenario evolutivo: los fósiles son incompletos en relación con los grupos actuales.

La matriz hipotética del caso de estudio 1 (Cuadro 1). Se utiliza el símbolo † para denotar la forma fósil hipotética en este caso.

La presencia de un taxón $(\mathrm{J})$ con siete caracteres codificados como "?" implica una posible serie de resoluciones falsas (Platnick et al. 1991). Estos arreglos pueden conducir a la postulación de hipótesis de filogenia falsas (Platnick et al. 1991, Retana-Salazar y Retana-Salazar 2008).

El análisis exhaustivo de la matriz completa obtiene 11 árboles óptimos $(\mathrm{L}=44, \mathrm{CI}=0,455$, $\mathrm{RI}=0,538)$. El consenso estricto de estas 11 topologías presenta la siguiente notación: $(\mathrm{H}(\mathrm{F}(\mathrm{C}(\mathrm{A}$, $\mathrm{D}, \mathrm{G}, \mathrm{J}(\mathrm{B}, \mathrm{E}, \mathrm{I}))))$ ).

Cuadro 1

Matriz hipotética para la codificación de un único taxón incompleto

\begin{tabular}{cc}
\hline A & 11111100011011001101 \\
B & 0100100110111111110 \\
C & 10101110101001101111 \\
D & 10111111111010011011 \\
E & 1111101101101111110 \\
F & 10100110001011101101 \\
G & 11101111111011011111 \\
H & 10110010101101110101 \\
I & 11011101111111111110 \\
$\mathrm{~J} \dagger$ & $? 01 ? ? 11 ? 11 ? ? 011 ? 1011$ \\
$\mathrm{X}$ & 00000000000000000000 \\
\hline
\end{tabular}

\section{Paso 1 del análisis}

Se analiza la matriz de datos excluyendo la forma fósil J. Se obtiene el siguiente resultado 24 árboles óptimos ( $\mathrm{L}=43, \mathrm{CI}=0,465, \mathrm{RI}=0,531)$. $\mathrm{El}$ consenso estricto de estas 24 topologías presenta la siguiente notación: (A,C,D,F,G,H,(B,E,I)).

Es importante acotar que en este caso la resolución baja al eliminar la forma fósil lo que indica que la misma es de alto valor en la determinación de los grupos filogenéticamente estables.

\section{Paso 2 del análisis}

Se analiza la matriz de datos incluyendo la forma fósil J y excluyendo los caracteres codificados como "?". Se obtiene el siguiente resultado 3 árboles óptimos ( $\mathrm{L}=29, \mathrm{CI}=0,448, \mathrm{RI}=0,543)$. $\mathrm{El}$ consenso estricto de estas 3 topologías presenta la siguiente notación: $(\mathrm{H}(\mathrm{F}(\mathrm{A}, \mathrm{C}, \mathrm{D}, \mathrm{G}, \mathrm{J},(\mathrm{B}, \mathrm{E}, \mathrm{I}))))$. Al analizar todas las topologías en conjunto se obtiene que la forma fósil J mantiene un $67 \%$ de probabilidad de mantener una posición basal al grupo $(\mathrm{D}, \mathrm{G}, \mathrm{A}(\mathrm{B}, \mathrm{E}, \mathrm{I}))$, por lo que la topología más probable es la mostrada en la figura 1 .

\section{Análisis del caso 2}

Escenario evolutivo: al menos un taxon fósil y uno actual se hallan incompletos.

La matriz hipotética del caso de estudio 2 (Cuadro 2). Se utiliza el símbolo $\uparrow$ para denotar las formas fósiles hipotéticas en este caso.

La presencia de un taxón fósil ( $\mathrm{J}$ ) con tres caracteres codificados como "?" implica una posible serie de resoluciones falsas, esto también sucede con los taxa A-I en los cuales no se hallan los caracteres 1-4 que se presentan en la forma fósil J (Platnick et al. 1991). Estos arreglos pueden conducir a la postulación de hipótesis de filogenia falsas (Platnick et al. 1991, Retana-Salazar y Retana-Salazar 2008).

El análisis exhaustivo de la matriz completa obtiene 4 árboles óptimos $(\mathrm{L}=37, \mathrm{CI}=0,514$, 


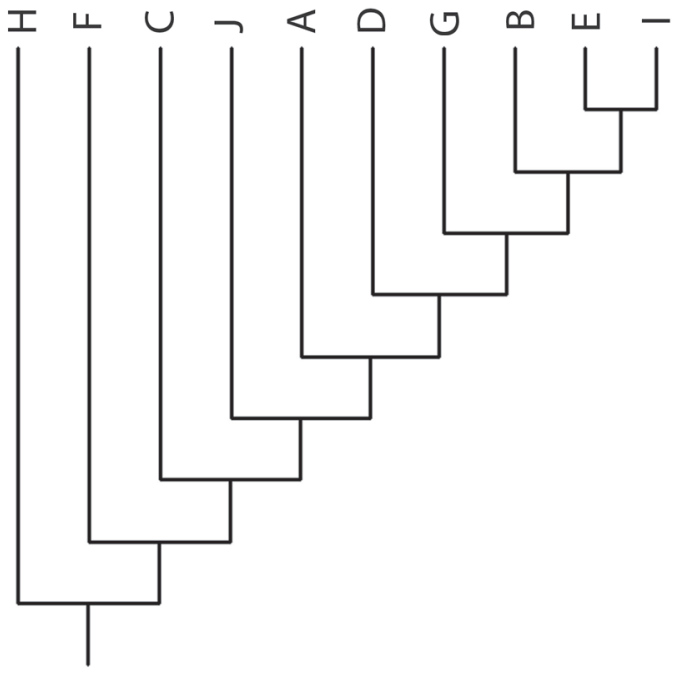

Fig. 1: Árbol filogenético con inserción de la forma fósil J en el punto de máxima probabilidad. Esta topología mantiene los estadísticos $\mathrm{L}=29, \mathrm{CI}=0,448, \mathrm{RI}=0,543$ ).

$\mathrm{RI}=0,581$ ). El consenso estricto de estas 4 topologías presenta la siguiente notación: $(\mathrm{A}, \mathrm{C}, \mathrm{F}, \mathrm{H}(\mathrm{D}, \mathrm{G}, \mathrm{J}(\mathrm{B}, \mathrm{E}, \mathrm{I})))$.

\section{Paso 1 del análisis}

Se analiza la matriz de datos excluyendo las forma fósil $\mathrm{J}$ y en consecuencia eliminando los caracteres 1-4 de la matriz de datos que se hallan presentes solo en la forma fósil. Se obtiene el siguiente resultado 2 árboles óptimos $(\mathrm{L}=31$, $\mathrm{CI}=0,516, \mathrm{RI}=0,605)$. El consenso estricto de estas 2 topologías presenta la siguiente notación: $(\mathrm{H}(\mathrm{F}(\mathrm{A}(\mathrm{C}((\mathrm{D}, \mathrm{G}),(\mathrm{B}, \mathrm{E}, \mathrm{I}))))))$.

Es importante acotar que en este caso la resolución aumenta al eliminar la forma fósil lo que indica que la misma introduce caracteres propios que alteran la topología de forma diferente que cuando solo se consideran las formas actuales. Este comportamiento sugiere que la ausencia de esta información en el análisis de filogenia elimina alternativas del posible proceso evolutivo que deben ser explicadas, por lo que la forma fósil es de valor en la inferencia del proceso evolutivo del grupo en estudio.
Cuadro 2

Matriz hipotética para la codificación donde al menos un taxon fósil y uno actual se hallan incompletos

\begin{tabular}{cc}
\hline $\mathrm{A}$ & $? ? ? ? 1100011011001101$ \\
$\mathrm{~B}$ & $? ? ? ? 1001101111111110$ \\
$\mathrm{C}$ & $? ? ? ? 1110101001101111$ \\
$\mathrm{D}$ & $? ? ? ? 1111111010011011$ \\
$\mathrm{E}$ & $? ? ? ? 1101101101111110$ \\
$\mathrm{~F}$ & $? ? ? ? 0110001011101101$ \\
$\mathrm{G}$ & $? ? ? ? 1111111011011111$ \\
$\mathrm{H}$ & $? ? ? ? 0010101101110101$ \\
$\mathrm{I}$ & $? ? ? ? 1101111111111110$ \\
$\mathrm{~J} \dagger$ & $10110111111101 ? ? ? 011$ \\
$\mathrm{X}$ & 00000000000000000000 \\
\hline
\end{tabular}

\section{Paso 2 del análisis}

Se analiza la matriz de datos incluyendo la forma fósil $\mathrm{J}$ y excluyendo los caracteres codificados como "?" tanto para las formas actuales como la fósil. Se obtiene el siguiente resultado 6 árboles óptimos ( $\mathrm{L}=28, \mathrm{CI}=0,464, \mathrm{RI}=0,583)$. $\mathrm{El}$ consenso estricto de estas 6 topologías presenta la siguiente notación: $(\mathrm{H}(\mathrm{F}(\mathrm{A}(\mathrm{C}(\mathrm{D}, \mathrm{G}, \mathrm{J},(\mathrm{B}, \mathrm{E}, \mathrm{I}))))))$. $\mathrm{Al}$ analizar todas las topologías en conjunto se obtiene que la forma fósil J mantiene un $67 \%$ de probabilidad de aparecer como adelphotaxon del $\mathrm{D}$, mientras que en la tricotomía (BEI) existe un $83 \%$ de asociación de (BE) y G mantiene la posición de adelphotaxon del grupo ((DJ)(I(BE))), por lo que la topología más probable es la mostrada en la figura 2 .

\section{Análisis del caso 3}

Escenario evolutivo: se incorporan varias formas fósiles por lo que es de importancia determinar cuales de ellas pueden indicar momentos de diversificación o momento de aparición del grupo estudiado. Los fósiles son incompletos en relación con los grupos actuales.

La matriz hipotética del caso de estudio 3 (Cuadro 3). Se utiliza el símbolo $\dagger$ para denotar las formas fósiles hipotéticas en este caso. 


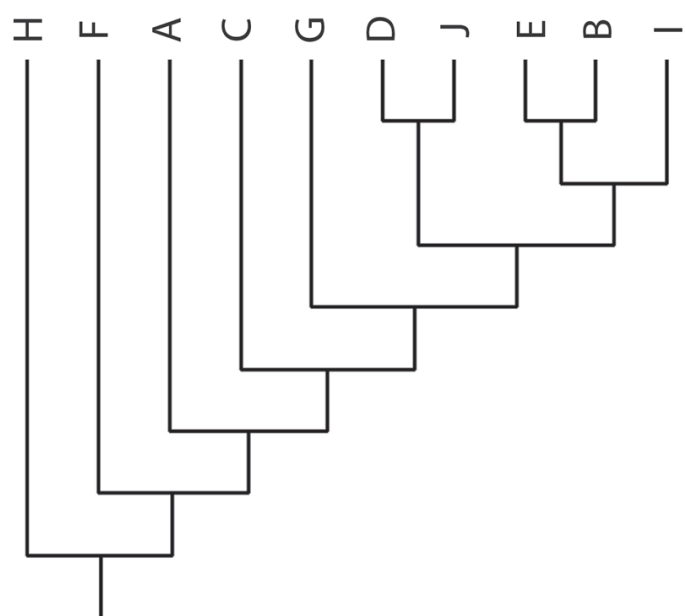

Fig. 2: Árbol filogenético con inserción de la forma fósil J en el punto de máxima probabilidad. Esta topología mantiene los estadísticos $\mathrm{L}=28, \mathrm{CI}=0,464, \mathrm{RI}=0,583$.

La presencia de los taxa fósiles $(\mathrm{H}, \mathrm{I}, \mathrm{J})$ con 7 y 9 nueve caracteres codificados como "?” implica una serie de resoluciones falsas (Platnick et al. 1991). Estos arreglos, al igual que en los casos anteriores, pueden conducir a la postulación de hipótesis de filogenia falsas (Platnick et al. 1991, Retana-Salazar y Retana-Salazar 2008).

El análisis exhaustivo de la matriz completa obtiene 3 árboles óptimos $(\mathrm{L}=41, \mathrm{CI}=0,488$, $\mathrm{RI}=0,543)$. El consenso estricto de estas 3 topologías presenta la siguiente notación: (A,C,F,H,D,G,J(B,E,I)).

\section{Paso 1 del análisis}

Se analiza la matriz de datos excluyendo las formas fósiles H, I, J. Del análisis se obtiene el siguiente resultado 1 árbol óptimo $(\mathrm{L}=36$, $\mathrm{CI}=0,556, \mathrm{RI}=0,529)$. La presencia de una única resolución elimina la posibilidad y la necesidad de un consenso estricto o de cualquier otro tipo, ya que es necesario al menos dos resoluciones para poder establecer un consenso. La obtención de un único árbol óptimo indica la presencia de una matriz altamente resuelta en cuanto a la codificación de los caracteres para las formas actuales consideradas. Esta topología presenta la siguiente notación: $(\mathrm{A}(\mathrm{F}(\mathrm{C}((\mathrm{B}, \mathrm{D})(\mathrm{D}, \mathrm{G})))))$.
Como era de esperar la resolución aumenta al eliminar las formas fósiles, ya que elimina las formas más incompletas del análisis. Este comportamiento sugiere que la ausencia de esta información en el análisis de filogenia elimina alternativas del posible proceso evolutivo que deben ser explicadas.

\section{Paso 2 del análisis}

Se analiza la matriz de datos incluyendo las formas fósiles más completas ya que en teoría presentan mayor información que permite minimizar la posibilidad de error al ubicarlas dentro de la filogenia, en este caso I-J y excluyendo los caracteres codificados como "?". Se obtiene el siguiente resultado 78 árboles óptimos $(\mathrm{L}=28$, $\mathrm{CI}=0,464, \mathrm{RI}=0,516)$. El consenso estricto de estas 78 topologías presenta la siguiente notación: (F,A,C,D,G, J,B,E,I). En este caso el árbol consenso no ayuda en la determinación de la posición de las formas fósiles, pero el cálculo del consenso de mayoría o de Nelson, indica que las posiciones más probables serían (BI) con un 55\% de probabilidad y la politomía (ACDFGJ) con un 64\% de probabilidad, la topología general es descrita por la notación: (E(BI)(ACDFGJ)). Las formas I-J mantienen una codificación similar en cuanto a

Cuadro 3

Matriz hipotética para la codificación donde los fósiles son incompletos en relación con los grupos actuales

\begin{tabular}{cc}
\hline $\mathrm{A}$ & 11111100011011001101 \\
$\mathrm{~B}$ & 01001001101111111110 \\
$\mathrm{C}$ & 10101110101001101111 \\
$\mathrm{D}$ & 10111111111010011011 \\
$\mathrm{E}$ & 11111101101101111110 \\
$\mathrm{~F}$ & 10100110001011101101 \\
$\mathrm{G}$ & 11101111111011011111 \\
$\mathrm{H} \dagger$ & $1 ? 1 ? ? ? 1 ? ? ? 1101 ? 1010 ?$ \\
$\mathrm{I} \dagger$ & $? 10 ? ? 10 ? 11 ? ? 111 ? 1110$ \\
$\mathrm{~J} \dagger$ & $? 01 ? ? 11 ? 11 ? ? 011 ? 1011$ \\
$\mathrm{X}$ & 00000000000000000000 \\
\hline
\end{tabular}


los caracteres codificados como "?" pero no comparten los mismos caracteres estructurales, por lo que $\mathrm{J}$ se halla más cercano al grupo politómico que a la especie B.

En un siguiente paso se eliminan las formas I-J y se inserta la forma $\mathrm{H}$, excluyendo los caracteres codificados como "?". Se obtiene el siguiente resultado 23 árboles óptimos $(\mathrm{L}=21$, $\mathrm{CI}=0.524, \mathrm{RI}=0.474)$. El consenso estricto de las 23 topologías presenta la siguiente notación: (A,B,C,D,E,F,G,H). El consenso de mayoría establece que la relación de mayor probabilidad es (BE) con un 57\% de probabilidad, esto contrasta con el resultado del posicionamiento de los fósiles I-J donde es más probable la relación (BI), considerando ambos casos el grupo más probable será $(\mathrm{E}(\mathrm{BI}))$ dado que los fósiles I-J son más completos que el fósil $\mathrm{H}$. En este caso el posicionamiento de los fósiles I-J no se hallan en igual posición debido a sus demás caracteres, de esta forma I es un fósil que indica diversificación, en este caso una especie de gran derivación que se extinguió lo que da una idea de la antigüedad de las especie B que aún persiste siendo su adelphotaxon. Mientras que H-J presentan igual grado de información a pesar de que $\mathrm{J}$ es más completo que $\mathrm{H}$. Esto evidencia que la creencia de que el estado de conservación (por ende lo completo del fósil) lo hace más o menos informativo no obedece a una realidad en su totalidad (Bravo-Cuevas y González-Rodríguez 2007). La notación de esta resolución sería (A,B,C,D,F,G,J,H (E(B,I)). Utilizando un criterio geológico se puede establecer que los grupos fósiles $\mathrm{H}-\mathrm{J}$ son basales a los taxa $(\mathrm{A}, \mathrm{B}, \mathrm{C}, \mathrm{D}, \mathrm{F}, \mathrm{G})$ presentando la siguiente notación $(\mathrm{H}, \mathrm{J}(\mathrm{A}, \mathrm{B}, \mathrm{C}, \mathrm{D}, \mathrm{F}, \mathrm{G},(\mathrm{E}(\mathrm{B}, \mathrm{I})))$.

\section{Un ejemplo concreto}

El caso de los grupos genéricos de Thripina. Los especialistas en Thysanoptera, grupo de insectos de pequeña talla de distribución, en su mayor parte, neotropical (Mound y Marullo 1996,
Retana-Salazar 2007), consideran que el grupo presenta serios problemas en su clasificación debido a la plasticidad registrada en las formas actuales, lo que dificulta los estudios de las relaciones filogenéticas dentro del grupo por el alto nivel de homoplasia registrado (Gauld y Mound 1982, Mound 1994, Retana-Salazar 1998), lo cual dificulta que la clasificación obedezca a verdaderos grupos naturales (Retana-Salazar y SotoRodríguez 2001, Retana-Salazar 2007).

La familia Thripidae es en este contexto una de las más complejas por su alta variabilidad (Bhatti 2006) y la misma ha sido dividida en múltiples familias en los últimos años (Bhatti 2006, Bhatti y Borbón 2008). Dentro de este grupo Mound y Palmer (1980) revisan una serie de géneros de la subtribu Thripina con lo que definen seis grupos de géneros en base al análisis de ocho caracteres morfológicos.

A partir de material fósil del Mioceno se describe un nuevo thripino para el Norte de España (Peñalver, 1998). El Dr. Enrique Peñalver lo ubica por sus características morfológicas dentro de Thripidae y en la subfamilia Thripinae por la ausencia de ornamentación reticular. Sin embargo, el estudio de las ilustraciones y fotos del material indican que este material fósil presenta al menos un par de setas posteroangulares largas en el pronoto, lo que ubica a esta forma fósil dentro de la subtribu Thripina.

Se ha tomado esta forma fósil para ejemplificar la inclusión de fósiles dentro de grupos actuales de especies, utilizando los grupos propuestos por Mound y Palmer (1981) como también los caracteres utilizados por estos autores para definir los grupos de géneros.

En el cuadro 4 se observa la matriz de caracteres para la subtribu Thripina según lo definen Mound \& Palmer (1981). Se utiliza el símbolo † para denotar la formas fósil del Mioceno Español, descrita por Peñalver (1998).

En el cuadro 5 se expresan los caracteres propuestos por Mound y Palmer (1981), con sus valores de CI y RI para la reconstrucción filogenética 
de mayor probabilidad para el fósil. Abreviaciones en el cuadro apo=apomorfía, ples $=$ plesiomorfía, $(-$ )$=$ ausente,$(+)=$ presente, $\mathrm{a}=$ anterior, $\mathrm{p}=$ posterior, $\mathrm{r}=$ reducido, $\mathrm{d}=$ desarrollado, $\mathrm{i}=$ incompleto, $\mathrm{c}=$ completo.

La presencia del taxón fósil presenta los caracteres 2 y 4 codificados como "?" esto implica la presencia de resoluciones falsas (Platnick et al. 1991). Estos arreglos, pueden conducir a la postulación de hipótesis de filogenia falsas (Platnick et al. 1991, Retana-Salazar y Retana-Salazar 2008).

El análisis exhaustivo de la matriz completa obtiene 27 árboles óptimos $(\mathrm{L}=12, \mathrm{CI}=0,750$, $\mathrm{RI}=0,571)$. El consenso estricto de estas 27 topologías presenta la siguiente notación: (Frankliniella gr, Megalurothrips gr, Mycterothrips gr, Taeniothrips gr, Fósil gr (Dorcadothrips gr, Thrips gr)).

\section{Paso 1 del análisis}

Se analiza la matriz de datos excluyendo la forma fósil. Del análisis se obtiene el siguiente resultado 6 árboles óptimos $(\mathrm{L}=11, \mathrm{CI}=0,727$, $\mathrm{RI}=0,571)$. El consenso estricto de estas 6 topologías presenta la siguiente notación: (Frankliniella gr, Megalurothrips gr, Mycterothrips gr, Taeniothrips gr, Fósil gr (Dorcadothrips gr,

Cuadro 4

Matriz de caracteres para la subtribu Thripina, definida por Mound \& Palmer (1981)

\begin{tabular}{cc}
\hline Dorcadothrips gr & $1110110(0 \& 1)$ \\
Frankliniella gr & 10210010 \\
Megalurothrips gr & 10111000 \\
Mycterothrips gr & 11111000 \\
Taeniothrips gr & 11110000 \\
Thrips gr & $(1 \& 2) 110(0 \& 1) 01(0 \& 1)$ \\
Fosil† & $2 ? 1 ? 0000$ \\
Ancestro & 00000000 \\
\hline
\end{tabular}

Thrips gr)). Este consenso coincide en este caso con la anterior topología utilizando toda la matriz de datos.

Como era de esperar la resolución aumenta al eliminar las formas fósiles, ya que elimina las formas más incompletas del análisis, esto se evidencia al obtener solo 6 árboles óptimos, 21 menos que en el análisis anterior. Este comportamiento sugiere que la ausencia de esta información en el análisis de filogenia elimina alternativas del posible proceso evolutivo que debe ser explicadas.

\section{Paso 2 del análisis}

Se analiza la matriz de datos incluyendo la forma fósil excluyendo los caracteres codificados como “?”. Se obtiene el siguiente resultado 2 árboles óptimos ( $L=7, C I=1,00, R I=1,00)$. El consenso estricto de estas 2 topologías presenta la siguiente notación: (Taeniothrips gr, Fósil (Frankliniella gr, Thrips gr)(Dorcadothrips gr, Megalurothrips gr, Mycterothrips gr)). El criterio del árbol consenso en este caso muestra a la forma fósil como politómica con Taeniothrips gr en la base de Thripina, un criterio de temporalidad geológica indica que este grupo debe ser anterior en tiempo y espacio a Taeniothrips gr como se muestra en la figura 3. En esta resolución existe una congruencia del 100\% para la resolución de Frankliniella gr y Thrips gr como para Dorcadothrips gr, Megalurothrips gr y Mycterothrips gr. Con esto se estima de forma consistente la posición de los grupos fósiles dentro de la filogenia establecida a partir de las formas actuales, ayudando a obtener una hipótesis de filogenia más exacta y con mejores criterios para la propuesta de nuevas posibles alternativas de clasificación naturales.

La resolución de esta filogenia a partir de los caracteres estudiados por Mound y Palmer (1980) identifica que la forma fósil descrita por Peñalver (1998) es un grupo de particular importancia en la definición de la subtribu Thripina, sobre la cual ha habido una seria discusión durante los últimos 
Cuadro 5

Caracteres propuestos por Mound y Palmer (1981) para la reconstrucción filogenética de mayor probabilidad

\begin{tabular}{cccccc}
\hline Carácter & & CI & RI & apo & ples \\
\hline 1 & número de antenómeros & 1.00 & 0.0 & $7-8$ & 9 \\
2 & seta preocelares & 0.50 & 0.0 & - & + \\
3 & setotaxia del pronoto & 1.00 & 0.0 & $\mathrm{r}$ & $\mathrm{d}$ \\
4 & par de setas medias del metanoto & 0.50 & 0.0 & $\mathrm{a}$ & $\mathrm{p}$ \\
5 & setotaxia de la vena I del ala I & 1.00 & 1.00 & $\mathrm{i}$ & $\mathrm{c}$ \\
6 & par medio de la setas tergales del abdomen & 1.00 & 0.0 & $\mathrm{r}$ & $\mathrm{d}$ \\
7 & ctenidia de los tergos abdominales & 1.00 & 1.00 & + & - \\
8 & setas discales esternales y pleurotergales & 1.00 & 0.0 & - & + \\
\hline
\end{tabular}

30 años en la literatura, acerca de la validez de los caracteres que la definen y en consecuencia de la validez taxonómica de este grupo. La posición de este fósil parece indicar que se trata de un grupo muy consistente y en el cual cuando se eliminan los caracteres que no son observables en los fósiles obtenemos una hipótesis de filogenia altamente resuelta con una alta consistencia y una muy alta distribución de caracteres de importancia filogenética. Estos resultados indican que los

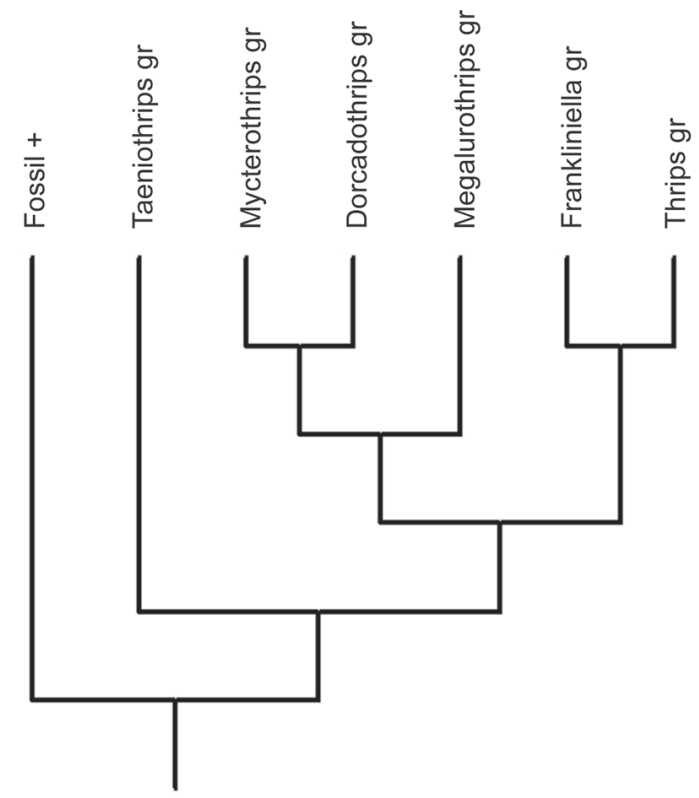

Fig. 3: Árbol fillogenético con inserción de la forma fósil descrita para el Mioceno (Peñalver 1998) en el punto de máxima probabilidad. Esta topología mantiene los estadísticos $\mathrm{L}=7$, $\mathrm{CI}=1,00, \mathrm{RI}=1,00$. caracteres propuestos por Mound y Palmer (1980) en efecto contienen una alta información filogenética, la cual es fuertemente respaldada por los hallazgos paleontológicos recientes. En consecuencia, la presencia de setas largas en el borde posterior del pronoto es una buena característica para la segregación de este grupo, carácter que se observa en los ejemplares de este grupo de especies desde el Mioceno.

Los resultados obtenidos indican con claridad que la forma fósil hallada y descrita por Peñalver (1998) merece ser considerada como un género aparte y el cual merece un grupo genérico aparte de gran valor en la determinación jerárquica de la subtribu Thripina. Este fósil parece indicar el momento de diversificación de los Thripina que parece coincidir con el Mioceno y en la región Europea. La posición basal con respecto a Taeniothrips indica que este grupo de gran diversidad en regiones templadas es uno de los primeros descendientes de este linaje.

\section{DISCUSIÓN Y CONCLUSIÓN}

Las formas fósiles son altamente informativas de los procesos evolutivos. Sin embargo, son habitualmente ignoradas por los biólogos que trabajan en la determinación de filogenias como en la propuesta de nuevas hipótesis de clasificación (Sour-Tovar \& Montellano-Ballesteros 1994).

En las últimas décadas esta discusión ha planteado que las formas fósiles son de particular interés (Forey 1994, Moreno et al. 2007), pero las 
limitaciones de los métodos establecidos para el estudio de la filogenia (Platnick et al. 1991), como las mismas limitaciones de los caracteres que son observables en las formas fósiles hacen poco consistente la ubicación de los fósiles en las filogenias de las formas de vida actuales (Forey 1994).

Uno de los puntos de más relevancia es la consideración de que los fósiles si son muy incompletos son de poca utilidad, por la ausencia de una gran cantidad de caracteres que imposibilitan la correcta ubicación de los mismos en las hipótesis de filogenia actuales (Forey 1994, Bravo-Cuevas y González-Rodríguez 2007). Por otra parte, entre las utilidades prácticas del registro fósil está la calibración de metodologías de reconstrucción filogenética, utilizando grupos con un registro fósil amplio que permita evaluar los procesos evolutivos en forma comparativa con los métodos actuales (Retana-Salazar y Retana-Salazar 2008).

En el método propuesto en este trabajo, se establece que puede optimizarse la forma de calcular cual es la posición más certera de las formas fósiles en la filogenia. Uno de los grandes vacíos con respecto a la inclusión de los fósiles en las actuales filogenias, es la ausencia de caracteres que obligan a una codificación muy abundante en "?" como en símbolos de "no aplica" (Forey 1994). Estas codificaciones son altamente problemáticas en las resoluciones filogenéticas como ha sido establecido en la literatura a través de diversos estudios al respecto (Platnick et al. 1991, Arnedo 1999, Retana-Salazar y RetanaSalazar 2008). Estos problemas han evitado que la evidencia fósil sea más utilizada en las filogenias actuales.

Nelson (1978) considera que los fósiles son formas de vida extintas e incompletas, que deben someterse a análisis y no se puede partir del supuesto de que toda forma fósil es necesariamente ancestral a formas de vida que si se hallan presentes en la actualidad. En este sentido este autor tiene un argumento de mucho peso, ya que la presunción de ancestría por parte de los paleontólogos ha tenido que ser corregida múltiples veces. Esto es una consecuencia de la ausencia de buenos métodos de resolución con la inclusión de formas fósiles.

Otro problema derivado de esto es la consideración a priori de que los caracteres registrados en los fósiles son ancestrales y que los paleontólogos con gran frecuencia estiman la consecución de las separaciones filogenéticas en función de la posición estratigráfica de los fósiles hallados (Retana-Salazar y Retana-Salazar 2008). Esto en realidad introduce una serie de supuestos que llevan a errores de interpretación muy serios como que desprecia la posibilidad de que una forma fósil hallada en un determinado periodo geológico puede haber existido en periodos anteriores y que simplemente no se han hallado evidencias anteriores de su fosilización o no se dieron condiciones de fosilización en momentos anteriores o el ambiente en que se desarrollaba no era favorable a la fosilización. Despreciar estas posibilidades e inferir la cladogenesis en función de la estratigrafía es un error común y muy grave, este error se registra en la literatura de Hennig (1966).

La evidencia indica que las formas fósiles son de gran utilidad en la interpretación de los procesos evolutivos y deben ser fundamentales en la determinación de las jerarquías de clasificación (Forey 1994). Se han propuesto una serie de posibilidades para que la clasificación incluya a los fósiles (Forey 1994).

El presente método muestra como la resolución por partes del cladograma, resolviendo la hipótesis de filogenia en una primera etapa con las formas actuales por ser las que muestran el mayor número de caracteres observables para establecer las comparaciones, para luego proceder a un inclusión de la forma fósil en una segunda etapa, donde el mayor problema es evitar los caracteres que producen falsos agrupamientos (Platnick et al. 1991). Estos caracteres deben ser eliminados de los análisis iniciales y deben estudiarse sobre la topología final obtenida sin ellos para evitar los sesgos producidos por estos (Retana-Salazar y Retana-Salazar 2008). Como se evidencia en el análisis presentado de dos variantes del escenario evolutivo en el que aparecen fósiles, siempre hay una disminución en la cantidad de resoluciones óptimas, con una mejora de los estadísticos de los árboles, que indiscutiblemente se verán afectados por la inclusión de un mayor o menor número de taxa como por los caracteres utilizados. Por otra parte, es evidente que un grupo en el cual aumente la cantidad de formas fósiles, cada vez presenta mayores problemas para la determinación de las 
posiciones filogenéticas más congruentes. Esto debido al incremento de caracteres no observables en cada una de las formas fósiles recuperadas.

Las formas fósiles correctamente insertadas en las hipótesis de filogenia de los grupos actuales permite estimar con mayor certeza la posible polaridad de los caracteres en una matriz de datos (Forey 1994), aunque esta suposición goza de aceptación también debe ser considerada la posibilidad de que los fósiles no necesariamente presentan condiciones plesiotípicas de los caracteres. Además caracteres que se presentan en la formas fósiles y las actuales pueden deberse a soluciones evolutivas de diferentes entornos biológicos, con lo que estos caracteres serían homoplásicos y en consecuencia considerarlos homólogos de los presentes en la formas actuales puede inducir serios errores en la resolución filogenética (RetanaSalazar y Retana-Salazar 2008).

Sin embargo, los fósiles nos indicarán con claridad una vez que sean correctamente insertados cuales son los momentos más probables de aparición de un grupo y cual es el momento de diversificación más reciente (Forey 1994). Estos dos factores evolutivo y ecológico son de gran valor en la interpretación de los grupos, como en la definición de su importancia ecológica y en conservación (Moreno et al. 2007). Estas evidencias indiscutiblemente son de gran valor en la determinación de los límites jerárquicos de los taxa.

El ejemplo presentado en este trabajo con los grupos genéricos de Thripina y la resolución filogenética de los mismos considerando los caracteres propuestos en la literatura, indica que el fósil descrito por Peñalver (1998) es fundamental en la determinación del momento de diversificación de la subtribu Thripina, ,Además establece que las dudas existentes sobre los caracteres que definen a esta categoría deben de ser eliminadas y que las especies conflictivas señaladas en la literatura (Mound y Palmer 1980) son posiblemente el resultado de adquisiciones o pérdidas posteriores que deben analizarse independientemente en cada género con el respectivo desarrollo de análisis filogenéticos.

El grupo hallado por Peñalver (1998) debe recibir un nuevo nombre genérico en función de su posición dentro de la filogenia y debe ser considerado como el grupo genérico basal del grupo de los Thripina, que define su momento de mayor diversificación a partir del Mioceno. Que este fósil se halle en el Norte de España, en la Región de Aragón, y su posición basal con respecto a un grupo de gran presencia neotropical conlleva la necesidad de evaluar las hipótesis de origen del grupo, ya que los taxónomos clásicos han inferido en función de la mayor diversidad de especies en el neotrópico que el origen del mismo es en esta región del mundo. Sin embargo, esto puede ser una consideración equivocada, al analizar la evidencia fósil. Es necesario efectuar mayores estudios de biogeografía cladista de este grupo que nos permita reconstruir con mayor claridad el proceso evolutivo del mismo.

\section{AGRADECIMIENTOS}

Al Dr. Enrique Peñalver Mollá, del Museo Geominero Instituto Geológico y Minero de España por la revisión del manuscrito como por facilitar numerosa literatura al respecto, a la Profesora Odalisca Breedy Shadid por la corrección del idioma inglés.

\section{REFERENCIAS}

ARNEDO M.A., 1999: Cladismo: La reconstrucción Filogenética basada en parsimonia.- Bol. SEA. 26: 57-84.

AX, P., 1987: The Phylogenetic System.- 340 págs. Jhon Wiley, Chichester.

BHATTI J.S., 2006: The classification of Terebrantia (Insecta) into families. Oriental Insects, 40: 339-375.

BHATTI J.S \& BORBÓN C.M., 2008: A new genus and species of thrips related to Psilothrips Hood 1927 living on the South American saltbush, Atriplex lampa Gill. ex Moq. (Chenopodiaceae), in Argentina (Terebrantia: Thripidae)-Thrips 6: 1-43 
BRAVO-CUEVAS V.M \& GONZÁLEZRODRÍGUEZ K.A., 2007: El potencial de la evidencia fósil en la reconstrucción filogenético. En: A. CONTRERASRAMOS, C. CUEVAS-CARDONA, I. GOYENECHEA \& U. ITURBE (Eds): La Sistemática base del conocimiento de la biodiversidad.- Ed. Universidad Autónoma del Estado de Hidalgo. México: 75-83.

BROOKS R.D. \& WILEY E.O., 1986: Evolution as Entropy.- 415 págs. John Wiley and Sons. USA.

DALGLEISH R.C., PALMA R.L., PRICE R.D. \& SMITH V.S., 2006: Fossil lice (Insecta: Phthiraptera) reconsidered.- Systematic Entomology. doi: 10.1111/j.13653113.2006.00342.x.

FOREY, P.L., HUMPHRIES, C.J., KITCHING, I.J., SCOTLAND, R.W., SIEBERT D.J. \& WILLIAMS, D.M., 1992: Cladistics.- 184 págs. Clarendon Press. Oxford. UK.

GAULD I.D. \& MOUND L.A., 1982: Homoplasy and the delineation of holophyletic genera in some insects groups. Syst. Entomol. 7:73-86.

GAUTHIER J., KLUGE A.G. \& ROWE T., 1988: Amniote phylogeny and the importance of fossils.- Cladistics, 4: 105-209.

HENNIG W., 1966: Phylogenetic systematics.284 págs. University of Illinois Press, Urbana, Illinois.

KITCHING I.J., 1994: Tree-building techniques.- In: FOREY, P.L., HUMPHRIES, C.J., KITCHING, I.J., SCOTLAND, R.W., SIEBERT D.J. \& WILLIAMS, D.M.(Eds.): Cladistics, Clarendon Press. Oxford. UK.: 48-64

LOTKA A.J., 1956: Elements of Mathematical Biology (2 ${ }^{\circ}$ Ed.).- 465 págs. Dover Books, New York.
MATTHEW W.D., 1926: The evolution of the horse: a record and its interpretations.Quart. Rev. Biol. 1:139-185.

MAYR E., 2001: What evolution is.- 318 págs. Basic Books, New York.

MORENO C.E., GIL G., SÁNCHEZROJAS G. \& ROJAS-MARTINEZ A.E., 2007: Conocimiento Sistemático para la Conservación Biológica.- En: CONTRERAS-RAMOS, A., CUEVASCARDONA, C., GOYENECHEA, I. \& ITURBE, U. (Eds.): La Sistemática base del conocimiento de la biodiversidad.- Ed. de la Universidad Autónoma del Estado de Hidalgo. México: 113-122.

MOUND L.A., 1994: Homoplasy and the systematics of phlaeothripine Thysanoptera, with a new short-tubed Australian Urothripine. Courier Forschunginstitut Senckenberg, 178: 21-25.

MOUND L.A. \& MARULLO R., 1996: The Thrips of Central and South America: An Introduction (Insecta: Thysanoptera).- Memoirs on Entomology International. 6: 487p.

MOUND L.A. \& PALMER J.M., 1981: Phylogenetic relationships between some genera of Thripidae (Thysanoptera).Entom. Scand. 15:153-170.

NELSON G.J., 1978: Ontogeny, phylogeny, paleontology, and the biogenetic law.- Syst. Zool. 27:324-345.

PATTERSON C., 1981: Significance of fossils in determining evolutionary relationships.Ann. Rev. Ecol. Systs. 12:195-223.

PEÑALVER-MOLLÁ E., 1998: Estudio tafonómico y paleoecológico de los insectos del Mioceno de Rábielos de Mora (Teruel).- 177 págs. Instituto de Estudios Turolenses, Excma. Diputación Provincial de Teruel, España. 
PLATNICK N.I., GRISWOLD C.E., \& CODDINGTON J.A., 1991: On missing entries in cladistics analysis.- Cladistics 7: 337-343.

RETANA-SALAZAR A.P., 2007: El grupo genérico Hoodothrips (Terebrantia: Heliothripidae).- Act. Zool. Lilloana, 51(1):,15-38.

RETANA-SALAZAR A.P., 1998: Una visión filogenética de Frankliniella (Thysanoptera: Thripidae).- Rev. Biol. Trop. 46(2):397-406.

RETANA-SALAZAR A.P \& RETANASALAZAR S.A., 2004: Hacia una lógica simple en la determinación de grupos biológicos: la especie y los grupos supraespecíficos (Forum).- Rev. Biol. Trop. 52(1): 19-26.

RETANA-SALAZAR A.P. \& RETANASALAZAR S.A., 2008: Entropía Biológica. Especies y Filogenia.- 167 págs. Editorial del Instituto Centroamericano para la Investigación en Biología y Conservación (ECIBRC), San José, Costa Rica.

RETANA-SALAZAR A.P. \& SOTORODRÍGUEZ G.A., 2001: Filogenia del género neotropical Zeugmatothrips (Thysanoptera: Phaleothripidae).- Gayana 65(2): 119-128.

SIMPSON G.G., 1951: Horses: the history of the horse family in the modern world and through sixty million years of history.- 384 págs. Oxford, University Press, New York.

SOUR-TOVAR F. \& MONTELLANOBALLESTEROS M., 1994: El concepto de especie en organismos fósiles. En: LLORENTE-BUSQUETS, J. \& LUNA, I.: Taxonomía Biológica.- Ed. de Universidad Autónoma de México, México:.450-498.

STIRTON R.A., 1940: The phylogeny of the North American Equidae.- Bull. Dept. Geol. Scs, University of California, 25:165-198. 